\title{
Fergus Sweeney
}

In March, the European Medicines Agency (EMA) launched its EU Clinical Trials Register. This new resource (www.clinicaltrialsregister.eu), which draws its information from the non-public EudraCT database, encompasses Phase I-IV paediatric trials and Phase II-IV adult trials that have at least one investigator site in Europe and all trials listed in a Paediatric Investigation Plan. Fergus Sweeney, Head of Compliance and Inspection Sector at the EMA, has been spearheading the development of EudraCT since the 1990s and has overseen the roll-out of the new public front. He spoke with

Asher Mullard about the past and future of this registry.

\begin{abstract}
Q What was the purpose of the older, non-public, EudraCT database?

EudraCT was launched in 2004, and was established by the clinical trials directive (Dir 2001/20/EC). It is a database of clinical trials that is only accessible to the national competent authorities of the EU member states, the EMA and the European Commission (EC). Its purpose was to facilitate communication between the member states, so that they could see what was happening with clinical trials across the community; because trials are authorized separately by each member state, it's important that they can see what is going on elsewhere.
\end{abstract}

\section{Q After the EC said that some of the} EudraCT data needed to be made publicly available, the EMA planned to launch the EU Clinical Trials Register in December 2009. What delayed the launch until March 2011?

We had first to await the publication of the guidelines describing the register, which were finalized in February 2009. Also, the original EudraCT database was set up to facilitate communication between the EU member states. In order to create a better platform for our new, more flexible system, we restructured the database. A lot of this isn't visible to the user. The migration of the data from the original structure also proved to be more complicated than we expected.

Q Who do you see as the key beneficiaries of this public database?

One of the objectives of the registry is to avoid redundant trials. Particularly in the case of children, there was a concern that researchers may be repeating procedures in different countries if they are not aware of what others are doing. Patients - particularly those with rare or life-threatening diseases - and their carers may also consider joining a clinical trial as one of their best treatment options. Therefore, there's an interest for patients and their health care providers to have a wider overview of research and what's being done where. There is also a broader interest in transparency across government and the EMA.

Q The World Health Organization (WHO) has set out 20 minimal criteria that must be met for registries to be included in the International Clinical Trials Registry Platform (ICTRP). Have you met these criteria?

As far as we're concerned we have mapped the fields that we made public against what the WHO has set out. We are already in discussion with the WHO about including our public information in the ICTRP.

\section{Q But aren't some of the fields mandated by} the WHO - including, for example, a contact point - currently empty for many trials in the EU Clinical Trials register?

Our structure includes the ability to display public contact information, but for older trials this information is not necessarily present yet. For instance, we only started asking sponsors to provide a public information access point in March 2010. We have written to industry associations to ask them to provide contact points for older trials.

If I went out tomorrow to build a public register from zero, I would build it differently, focusing more directly on those WHO fields and on a system in which sponsors would post their own information. But we have the system we have - we are constrained by the processes and the data structure that we have historically.
Q New legislation has also called for a 'results section' to be added to this register. How is this coming along?

Guidance on the 'results section' has to be completed, agreed and published by the EC, which I anticipate will happen by the end of this year. We then have to develop the software, which is currently planned to happen for the end of next year. But we'll have to see how other demands affect the development timeline of the project.

\section{Q What else might change with future launches?} I have two priorities overall. The first is to improve data quality - the completeness of the questions, their accuracy and the use of standardized terminology. Second, I want to improve the user experience. We need a better search function. This will come partly with having better data quality, but we are also looking at a number of tools that can improve our search function.

I would also hope that in a future release, within the next 18 months, sponsors will be able to maintain at least some of the data sets directly. And in the current version, users can't see historical versions of a protocol, but that is something that could change in the future. There are also possibilities of widening the scope of the system to perhaps include trials in earlier development and trials that are conducted wholly overseas. But many of these changes will be driven by legislative requirements.

Q How many new trials are likely to be entered into the public database each year? EudraCT receives around 2,500 new clinical trials each year. A little over a third of these are Phase I trials, so we're probably talking about approximately 1,500 trials a year that will go into the public domain. 